\title{
Using Nonlinear Ultrasound to Track Microstructural Changes due to Thermal Aging in Modified 9\%Cr Ferritic Martensitic Steel
}

\author{
Daniel Marino ${ }^{1}$, Jin-Yeon Kim ${ }^{1}$, Alberto Ruiz ${ }^{2}$, Young-Sang $\mathrm{Joo}^{3}$, Jianmin Qu ${ }^{4}$, and Laurence $\mathbf{J}$ \\ Jacobs $^{1}$ \\ 1. School of Civil and Environmental Engineering, Georgia Institute of Technology. Atlanta, GA 30332 \\ 2. Instituto de Investigación en Metalurgia y Materiales, U.M.S.N.H., México \\ 3. SFR NSSS System Design Division, Korea Atomic Energy Research Institute, Daejon, Korea
}

4. School of Civil and Environmental Engineering, Northwestern University

\begin{abstract}
This research investigates second harmonic generation in Rayleigh surface waves propagating in 9\%Cr ferritic martensitic steel. Previous experimental results show that nonlinear ultrasound is sensitive to certain microstructural changes in materials such as those due to thermal embrittlement and precipitation hardening. This research measures the ultrasonic nonlinearity parameter as an indicator of microstructural changes due to thermal aging in 9\%Cr ferritic martensitic steel specimens. The specimens are isothermally aged for different holding periods to induce progressive changes in the microstructure and to obtain different levels of thermal damage. As thermal aging progresses, the existing dislocations are annihilated in the beginning and precipitates are formed; these microstructural evolutions lead to large changes in the measured nonlinearity parameter, $\beta$. Nonlinear ultrasonic experiments are conducted for each specimen using a wedge transducer for generation and an air-coupled transducer for detection of Rayleigh surface waves. The amplitudes of the first and second order harmonics are measured as a function of propagation distance, and these amplitudes are used to obtain the relative nonlinearity parameter at different aging stages. A possible scenario for the microstructural evolution during thermal aging is proposed based on the results from the nonlinear ultrasonic measurements, scanning electron microscopy (SEM), and Rockwell HRC hardness. These results indicate a clear trend that the measured nonlinearity parameter is sensitive to variations in dislocation and precipitate density, and thus can be useful in tracking microstructural changes in this material during thermal aging.
\end{abstract}




\section{INTRODUCTION}

For decades, 9\%Cr steel has been an attractive solution for high temperature and pressure applications, since the material provides sufficient strength, resistance to corrosion and oxidation, low thermal expansion, and adequate fatigue resistance. Furthermore, 9\% $\mathrm{Cr}$ steel is relatively cheap as compared to other materials such as austenitic stainless steels which meet the same requirements [1]. However, an increased demand to improve the efficiency of power plants requires an exposure to higher temperatures and pressures, which may lead to drops in the abovementioned mechanical properties. The elevated temperature changes the microstructure as various precipitations respond differently to environmental conditions [1]. Thermal aging of ferritic martensitic steel at a temperature $600^{\circ} \mathrm{C}$ or higher can pose a high risk to components such as turbines and pressure vessels because of a drop in the mechanical properties of the steel, such as strength or creep resistance. For this reason, a more quantitative understanding of the microstructural evolution during thermal aging in this material has been of considerable interest to material engineers and scientists in the power plant industry.

The changes in the microstructure, e.g. the growth of pre-existing precipitation $\left(\mathrm{M}_{23} \mathrm{C}_{6}\right.$, MX, etc.) or the formation of Laves phase during thermal aging [2], and an accelerating dissolution of lath martensite [3] that contains a high amount of dislocations, cause a degradation of the steel's mechanical properties. The industry requires reliable methods to evaluate this thermal degradation, and ultrasonic nondestructive evaluation (NDE) has demonstrated its capabilities for assessing microstructural changes in different metallic materials. It is possible to use either linear ultrasonic or nonlinear ultrasonic methods to approach this problem.

Nonlinear ultrasonic NDE techniques are preferred over conventional linear ultrasonic techniques because of their superior ability to detect microstructural changes prior to significant drops in strength and failure. Higher harmonics are generated when ultrasonic waves interact with a material's microstructure [4] such as precipitates [5], microvoids [6], or radiation damage [7]. The nonlinearity parameter $\beta$ relates the amplitudes of the first and second harmonic waves, and contains information on the material's microstructure. Hikata et al. [8] derived a relationship between the pinning of dislocations and the generation of a second harmonic component. Cantrell et al. [9] extended this earlier study by considering the stress field introduced by precipitates, which pins dislocations. The findings of these studies and further 
experiments [3], [10] indicate the potential of nonlinear ultrasound to track microstructure changes due to thermal aging.

The use of nonlinear Rayleigh surface waves has the advantage of only requiring singlesided access, making them particularly suitable for field applications. Furthermore, Rayleigh waves can travel longer propagation distances than bulk waves due to less spreading. Most importantly, it is possible to effectively isolate the material contribution in the measured second harmonic component by varying the propagation distance; here the unwanted nonlinearity from the measurement system will remain constant (or decrease), while the material nonlinearity will increase with increasing propagation distance [10].

The objective of this research is to develop a nonlinear ultrasonic method to track changes in the microstructure of thermally aged $9 \% \mathrm{Cr}$ steel. A non-contact detection method provides the possibility to automate the measurement process. With the help of the measured nonlinearity parameter $\beta$, the state of the microstructure in this material is experimentally assessed. In addition, complementary measurements (hardness tests, metallurgical analyses) are performed to obtain additional information on the mechanical properties and microstructure of the aged steel to support the conclusions based on nonlinear ultrasonic results and to help interpret the trends observed in the measured $\beta$ values.

\section{THEORETICAL BACKGROUND}

\subsection{Nonlinearity Parameter}

The nonlinearity parameter for Rayleigh surface waves is similar to the nonlinearity parameter for longitudinal waves, because a Rayleigh wave can be described as a combination of a longitudinal and a shear wave component, and the contribution of the shear wave to the nonlinearity vanishes in an isotropic material. So, by considering a Rayleigh wave propagating in the $x_{1}$-direction with the $x_{3}$-axis pointing into the half-space, the acoustic nonlinearity parameter $\beta$ is given by [11]

$$
\beta=\frac{u_{3}(2 \omega)}{u_{3}^{2}(\omega)} \frac{8 i \kappa_{D}}{k_{D}^{2} k_{R}}\left(1-\frac{2 k_{R}^{2}}{k_{R}^{2}-\kappa_{S}}\right),
$$

where $u_{3}(\omega)$ and $u_{3}(2 \omega)$ are the out-of-plane displacement amplitudes for the first and second harmonics, respectively, and $\kappa_{D}=k^{2}{ }_{R}-k_{D}{ }_{D}$ and $\kappa_{S}=k^{2}{ }_{\mathrm{R}}-k_{S}{ }_{S}$, where $k_{D}, k_{S}$ 
and $k_{R}$ denote, respectively, the wavenumbers for the longitudinal, shear and Rayleigh waves in the material. In this study, the out-of-plane displacement amplitudes are measured for varying propagation distances. In this case, a relative acoustic nonlinearity parameter is defined as

$$
\beta^{\prime} \propto \frac{u_{3}(2 \omega)}{u_{3}^{2}(\omega) x_{1}}
$$

where the slope of the normalized ratio $u_{3}(2 \omega) / u_{3}^{2}(\omega)$ over the propagation distance $x_{1}$ is proportional to the acoustic nonlinearity parameter $\beta$.

The thermal aging process influences not only the dislocation density, but also the size of the precipitated particles; these are both the sources of material nonlinearity, and can also change the acoustic nonlinearity parameter. Based on the study of Hikata et al. [8], Cantrell et al. [12] derived an analytical model, which describes the interaction of dislocations and precipitated particles in metallic alloys. Suppose that the average distance of two pinning obstacles (precipitated particles) equals $2 L$, the radius of a precipitated particle $r_{p}$ can be related to the average distance and the precipitate volume fraction $f_{p}$ by

$$
L=\frac{2 r_{p}}{\sqrt[3]{f_{p}}} .
$$

Assuming that the dislocation density is low enough that the interaction among dislocations can be neglected, the acoustic nonlinearity parameter has the following form [12]

$$
\beta^{\text {dislocation-precipitation }} \propto \frac{\Lambda^{(d)} r_{p}^{4}}{\sqrt[3]{f_{p}}},
$$

where $\Lambda^{(\mathrm{d})}$ is the dislocation density. Note that the nonlinearity parameter theoretically depends: linearly on the dislocation density; the fourth power of the radius of precipitates; and reciprocally on the third root of the volume fraction of precipitates. The total acoustic nonlinearity parameter $\beta$ consists of contributions by the lattice and the interaction of dislocations and precipitations. If $\beta^{\text {lattice }}$ remains constant and $\beta^{\text {dislocation-precipitation }}$ changes during thermal aging, then the change in the total acoustic nonlinearity parameter $\Delta \beta$ provides information on the microstructural evolution.

\subsection{Microstructure Evolution during Thermal Aging}


In the material investigated, a modified 9\%Cr ferritic martensitic steel, heat treatment activates two mechanisms which occur simultaneously: the formation of precipitates and a change in the dislocation density. Both mechanisms influence the material's hardness and long-term properties, and thus the measured acoustic nonlinearity. In general, the precipitation process occurs in three stages; nucleation, growth, and coarsening.

Two different kinds of precipitates evolve during thermal aging in this maerial. First, there are the precipitates that evolve during tempering e.g., $\mathrm{M}_{23} \mathrm{C}_{6}, \mathrm{MX}$ and $\mathrm{M}_{2} \mathrm{X}$ particles, and second there are the Laves phase and Z-phase, which precipitate during further thermal aging. Cipolla et al. [13] and Hald et al. [14] conducted heat treatments on similar materials (amounts of some alloying elements are slightly different) to observe the microstructural evolution during thermal aging. Figure 1 shows the growth of these precipitates as a function of aging time Note that the evolution curves in this figure are produced by fitting the experimental data in [13] and [14]. The average radius of the $\mathrm{M}_{23} \mathrm{C}_{6}$ carbides is about $100 \mathrm{~nm}$ with a relatively high coarsening rate, but even after $10000 \mathrm{~h}$ at $650^{\circ} \mathrm{C}$, the critical radius of about $250 \mathrm{~nm}$ is not reached, as depicted in Figure 1 (green broken line). MX particles are very stable against coarsening, as illustrated in Figure 1 (blue dot-dashed line), and thus can be seen as the backbone of the longterm stability [15]. Laves phase is quite unstable at high temperatures (above $700^{\circ} \mathrm{C}$ ) [13], so it does not nucleate during tempering in the same way as the other precipitates. Laves phase forms during thermal aging and rapidly grows up to a size of about $400 \mathrm{~nm}$ in the first $1000-10000 \mathrm{~h}$ as depicted in Figure 1 (red solid line), and it has a long nucleation and growth phase [14]. Z-phase also precipitates during thermal aging, but it is not considered in this study since it occurs only for longer holding times.

FIGURE 1. Microstructural size evolution of M23C6 and Lave phase in 9\%Cr steel (curves are produced by fitting the experimental data in [13] and [14])

Dislocations are line defects in a crystal lattice that reduce the amount of free energy in the system. Consequently, regions with a high amount of dislocations are relatively unstable. It has been reported that not only the size of precipitates changes over aging time, but also the dislocation density decreases while tempering and aging [3,16]. According to the results of Sawada et al. [16] for a similar material, the initial dislocation density after normalizing at $19 \cdot 10^{14} \mathrm{~m}^{2}$ steadily diminishes to $6.1 \cdot 10^{14} \mathrm{~m}^{2}$ after tempering and $1.6 \cdot 10^{14} \mathrm{~m}^{2}$ after aging. 
TABLE 1. Microstructural changes and their effects on $\beta$.

Moreover, Park et al. [3] show that the lath martensite, containing a high dislocation density, dissolves into sub-grains after thermal aging. Consequently, the dislocation density quickly declines in the beginning, since more martensite is transformed, and after a certain time the dislocation density reaches an equilibrium state. The expected influence of the resulting mechanisms during thermal aging on $\beta$, according to the previously introduced Equation (4), are summarized in Table 1.

\section{MATERIALS AND METHODS}

\subsection{Specimen Preparation}

The modified 9\%Cr steel specimens are cut from a 0.5 inch thick plate that has a nominal yield strength of $620 \mathrm{MPa}$. The dimensions of the rectangular specimens are $203.2 \times 45.72 \times 12.7 \mathrm{~mm}^{3}$ in length, width, and thickness, respectively. The width and thickness are large enough so that the direct propagation of the Rayleigh waves are not disturbed by the waves reflected from the sides and bottom.

Table 2 shows the chemical composition of the 9\%Cr steel. As can be seen from Table 2, chromium (Cr) is the main alloying element since it gives a significant level of hot corrosion resistance, and also is essential to ensure high temperature oxidation resistance. In order to study the effect of temperature and time on the measured acosutic nonlinearity parameter, $\beta$, the specimens were isothermally heat treated at $650^{\circ} \mathrm{C}$ for $0 \mathrm{~h}, 200 \mathrm{~h}, 500 \mathrm{~h}, 1000 \mathrm{~h}, 1500 \mathrm{~h}$ or 3000 h, respectively.

Table 2. Chemical composition of $9 \%$ Cr steel

The small intervals between exposure times in the beginning are selected so as to not miss changes in the rapidly changing microstructure in the early stages. After the heat treatment, 
the surfaces were carefully ground by hand with grade 1000 emery paper to remove the oxidation layer produced during the heat treatment. In addition, to observe changes in the microstructure, a set of samples treated with the same conditions were prepared by conventional metallographic polishing and etching. Also, Rockwell C hardness measurements were performed to determine microstructural changes during the annealing process and to monitor the effects of the thermal treatments on the ferrite transformation process.

\subsection{Nonlinear and Linear Ultrasonic Measurements}

Figure 2 shows the experimental setup to generate and detect the nonlinear Rayleigh waves consisting of an air-coupled transducer, centered at $4 \mathrm{MHz}$, and a narrow band contact piezoelectric transducer (center frequency 2.25 MHz), which excites a longitudinal wave on a wedge.

FIGURE2. Experimental setup for nonlinear ultrasound measurements.

The generation transducer is coupled to an acrylic wedge to excite Rayleigh waves in the specimen. A function generator is used to introduce a tone burst signal with a peak-to-peak voltage of $800 \mathrm{mV}$ at $2.1 \mathrm{MHz}$. To ensure a sufficiently long steady state portion for the subsequent signal processing, the generation signal contains 20 cycles. To measure second harmonic waves in the specimen, a RITEC GA-2500A is employed which amplifies the output signal of the function generator by as much as $60 \mathrm{~dB}$. The high-amplitude Rayleigh waves travel along the surface and the air-coupled transducer receives the leaky Rayleigh surface waves, the acoustic waves leaked from the surface into the air. Therefore, the leaked Rayleigh waves have the same information as the Rayleigh surface waves in the solid specimen. The received signals are post amplified and then signal averaged 256 times to improve the signal-to-noise ratio (SNR).. The signals are recorded on an oscilloscope with a sampling rate of $250 \mathrm{MS} / \mathrm{s}$. Figure 3(a) shows a typical example of a time domain signal with a steady state portion in the middle. This time signal was taken at a distance of $25 \mathrm{~mm}$ from the front of the wedge transducer. A Hann window [17] is applied to suppress the transient responses in the beginning (voltage overshoot) and at the end (ringing voltage). The Fast Fourier transform (FFT) maps the time domain signal to the frequency domain. Figure 3(b) depicts the frequency spectrum 
(a)

(b)

corresponding to the time domain signal in Figure 3(a), where the contribution of the fundamental $A_{1}$ and second harmonic $A_{2}$ amplitudes are clearly visible. The amplitudes $A_{1}$ and $\mathrm{A}_{2}$ are the measured electrical output signals from the air-coupled transducer and are proportional to (but not equal to) the absolute out-of-plane displacement amplitudes $u_{3}(\omega)$ and $u_{3}(2 \omega)$ given in Eqs (1) and (2). Thus, the ratio $\mathrm{A}_{2} / \mathrm{A}^{2}$ is proportional to the acoustic nonlinearity parameter, $\beta$.

FIGURE3. Typical output signal in and frequency domain with $\mathrm{A}_{1}$ and $\mathrm{A}_{2}$

The wedge transducer that generates Rayleigh waves in the specimen is held at a fixed position and the air-coupled transducer moves, without contacting the specimen surface, by incremenets of $2 \mathrm{~mm}$ along the propagation path starting at $x_{1}=25 \mathrm{~mm}$ and ending at $x_{1}=71 \mathrm{~mm}$. Thiele et al. [10] describe the assumptions and specify the reasons to measure the acoustic nonlinearity in this fashion using a varying propagation distance. As previously observed $[10,18]$, a determination of the acoustic beam path has to be carried out beforehand, to find the actual propagation direction of the Rayleigh wave beam since it has been observed that the acoustic beam does not come from the center of the wedge, nor propagate in a direction normal to the excitation front; the beam typically propagates with an angle less than 1 deg with respect to the normal. To obtain comparable results and guarantee repeatability, an initial experiment to determine the acoustic path by moving the air-coupled transducer in the $x_{1}-x_{2}$ plane is necessary. Once the actual path is established, nonlinear measurements are performed along this predetermined path. At least 25 measurements for each specimen are performed; one measurement consists of the determination of the acoustic beam path and the nonlinear measurement along the predetermined propagation path. (b)

As shown in Figure 4(a), $A_{1}$ decreases monotonically with increasing propagation distance due to the diffraction and attenuation of the acoustic beam [18, 19]. Furthermore, the nonlinear interaction leads to a monotonic increase of $\mathrm{A}_{2}$ for short propagation distances, and then levels off due to attenuation and diffraction as noted in $[18,19]$. Figure 4(b) shows the ratio $\mathrm{A}_{2} / \mathrm{A}^{2}$, where the slope of the linear fit is proportional to the acoustic nonlinearity parameter $\beta$ in this small propagation distance $[10,20]$.

FIGURE 4. Typical results for a measurement with a frequency of $2.1 \mathrm{MHz}$ for sample 1 (untreated sample) 


\subsection{Velocity and Attenuation Measurements}

The velocity and attenuation coefficients of a longitudinal wave are measured for the same set of 9\%Cr steel specimens following the procedure of Ruiz et al. [21] using a $5 \mathrm{MHz}$ normal incidence longitudinal wave in an inmersion tank.

\section{EXPERIMENTAL RESULTS}

\subsection{Nonlinear Ultrasonic Results}

Figure 5(a) summarizes the nonlinear ultrasonic measurements conducted in this research. The normalized nonlinearity parameter $\beta$ is plotted with respect to the aging time in hours. The measured values of the acoustic nonlinearity parameter $\beta$ are normalized by the mean value measured in the untreated specimen. The error bars show the distribution of the measured results from all the specimens with the range representing the highest and lowest measured values; these error bars indicate the maximum variation of the experimentally measured results for each specimen. The maximum error was $10 \%$ for the last specimen, which still looks acceptable since the trend is clearly recognizable. Figure 5(a) shows an initially rapid decrease of $\beta$, followed by an increase after the aging time of $500 \mathrm{~h}$. In the first $500 \mathrm{~h}$, the acoustic nonlinearity parameter drops from $100 \%$ to about $70 \%$. This drop of $30 \%$ can be explained by the reduction of the dislocation density. The further increase until $3000 \mathrm{~h}$ is reasonable because the precipitated particles start to form and grow. At $3000 \mathrm{~h}$, the acoustic nonlinearity parameter $\beta$ reaches a value of $113 \%$. Due to these significant mesured changes in $\beta$, the conclusion can be drawn that the acoustic nonlinearity parameter is very sensitive to the microstructural evolution including changes in dislocations and precipitations occurring in this material.

FIGURE 5. Nonlinearity parameter $\beta$ (a) normalized by the mean value of specimen 1 and Rockwell C hardness (b) over holding time

This trend is supported by Park et al. [3] who also measure the nonlinearity parameter for a 10.5\%Cr steel with a different composition of alloying elements. This study investigates a 
similar material for several aging temperatures and their results for the nonlinearity parameter show a trend similar to that shown in Figure 5(a).

\subsection{Velocity and Attenuation}

Figure 6 shows the velocity and attenuation of longitudinal waves measured for the six different holding times.As it is seen in Figure 6, at $5 \mathrm{MHz}$, the material attenuation and velocity do not change significantly throughout the entire heat treatment. Figure 6 demonstrates that the measured linear ultrasonic parameters at this frequency are insensitive to the changes occuring in the microstructure being produced by the heat treatment.

FIGURE 6 Attenuation and velocity measurements as a function of holding time.

\subsection{Rockwell Hardness Test and Metallurgical Analysis}

A clear decrease of hardness with increasing holding time is observed in Figure 5(b) for the heat treated specimens. The modified $9 \% \mathrm{Cr}$ steel shows an initial, significant decrease up to a holding time of $500 \mathrm{~h}$. The average value drops from $22.5 \mathrm{HRC}$ to $19.8 \mathrm{HRC}$ within $500 \mathrm{~h}$. After that time, the hardness gradually reduces to a value of 18.5 HRC. A similar trend of rapid decrease, followed by a slight decrease in hardness is reported by Jones et al. [22] who investigated the behavior for different temperatures and found that with an increasing temperature, the initial slope becomes steeper. The trend reveals that the hardness is not the best indicator for thermal damage because there is no significant measurable change after $500 \mathrm{~h}$.

Scanning electron microscopy (SEM) is performed with a JOEL JSM-7600F microscope. Furthermore, element mapping and energy dispersive X-ray microanalyses (EDX) are conducted to emphasize the distribution and concentration of the three most important alloying elements: chromium, molybdenum and iron. To obtain the metallographic results, the specimens are carefully ground by hand to grade 1200 and subsequently polished with a $1 \mu \mathrm{m}$ diamond solution and cloth. The microstructure is revealed using a Villela's reagent, which is a common metallographic etchant for heat treated steels and martensitic stainless steels. It contains $100 \mathrm{~m} l$ ethanol, $5 \mathrm{ml} \mathrm{HCl}$ and $1 \mathrm{~g}$ of picric acid. Initially, particles are seen to be evenly distributed in the matrix and at the boundaries of grains. Both inter and intra granular precipitates of different morphologies are observed in the image, while most of them are aligned along the grain 
boundaries. Consider four typical SEM images captured at several different positions that are representative results of the microstructure. While Figure 7(a) shows very fine intra lath precipitates prior to the heat treatment with particle radii $r_{p}<<1 \mu \mathrm{m}$, Figure 7(c) shows the effect of the aging treatment as a carbide growing mechanism. It is noted that the precipitation is uniform and the density of precipitates is higher; furthermore, the micrographs show larger precipitates. As observed in Figures 7(b), iron (green) and molybdenum (blue) are homogeneously distributed, whereas a higher concentration of chromium (purple) shows up in some areas.

FIGURE 7. Element mapping of specimen with holding time of $0 \mathrm{~h}$ (left) and $3000 \mathrm{~h}$ (right).

However, it still has some level of regularity in its distribution. As aging time progresses, the diffusion of chromium is evident concentrating in the bigger particles as depicted in Figure 7(d).

Table 3 summarizes the results of the EDX at the particles and matrix indicated in Figures 7(a) and 7(c), where the element concentrations in the matrix (circle) and a particle $(P)$ are determined before and after the $3000 \mathrm{~h}$ heat treatment.

TABLE 3. Element concentration.

The chromium concentration after $3000 \mathrm{~h}$ holding time is four times higher than that in the untreated specimen. When the precipitated particle in the base material are compared with those after aging, the chromium content increses from $11.17 \%$ to $47.27 \%$, leading to a sharp decline of the iron concentration from $75.23 \%$ to $36.78 \%$. From Table 3, it can be seen that the carbon (8.8\%), chromium (11.17\%) and molybdenum (0.99\%) are richer in the precipitated particles than in the matrix. These results lead to the assumption that the particles may be a chromium carbide type. Furthermore, the particle size increases for each further aged specimen and after $3000 \mathrm{~h}$; some particles reach a size of about $0.8 \mu \mathrm{m}$ in diameter.

In general, the precipitation sequence depends mainly on the composition of the steel, but the diffusivities of the alloy elements and the ease of nucleation are also important parameters determining the favored carbide phases [22].

\subsection{Results and Discussion}


This section provides interpretations of the results from the nonlinear ultrasonic and complementary measurements. Figure 8 shows the qualitative trends of the dislocation density (dashed line) and the radius of precipitated particles (dotted line) that are extracted from other studies on similar steels ([3], [16] and [2], [13], [14] respectively), as well as the experimentally mesured acoustic nonlinearity and hardness. The entire behavior is divided into two phases: an initial phase which is dominated by decreasing contributions to $\beta$; followed by a second phase which is dominated by increasing contributions to $\beta$. The initial phase clearly shows a simultaneous decrease in the hardness and acoustic nonlinearity parameter. The decrease in nonlinearity up to $500 \mathrm{~h}$ is mainly caused by the reduction of the dislocation density $\Lambda^{(\mathrm{d})}$ and the precipitate nucleation process. The characteristics of the experimentally mesured $\beta$ and hardness allows for the conclusion to be drawn that the microstructural changes associated with the dropping dislocation density is the dominate mechanism. This phase where the value of $\beta$ decreases is followed by a phase where the growth of precipitated particles dominates, causing an increase in the acoustic nonlinearity parameter, $\beta$. The nonlinearity at the aging time of $3000 \mathrm{~h}$ exceeds even the starting value by approximately 13\%. In this increasing phase, the dislocation density changes very slowly, and simultaneously the previously nucleated particles grow in size. The results obtained in these measurements imply that the behavior after $500 \mathrm{~h}$ is probably dominated by the contribution of the growing precipitates. However, this hypothesis needs to be further investigated since the quantitative effects of dislocations and precipitated particles on $\beta$ are yet to be determined. Thus, a more precise investigation into the microstructural evolution is necessary through the determination of the dislocation density $\Lambda^{(d)}$, the exact size of the particles $r_{p}$ and their volume fraction $f_{p}$. Nonetheless, there is a significant change in $\beta$ over aging time in Figure 8, which allows an assessment of the stage of thermal aging. Note that the specimens show no signs of any other damage or cracks until $3000 \mathrm{~h}$.

FIGURE 8. Comparison of hardness and ultrasonic results with the qualitative trend of the dislocation density and radius of precipitated particles.

\section{CONCLUSION}


This research demonstrates that, compared with linear ultrasonic attenuation and velocity, the acoustic nonlinearity parameter $\beta$ is more sensitive to changes in the microstructure and $\beta$ is a useful parameter to track microstructural changes due to thermal aging in $9 \% \mathrm{Cr}$ ferritic martensitic steel. The nonlinear ultrasonic results indicate an initially rapid decrease, followed by an increase in the acoustic nonlinearity parameter $\beta$. The initial phase of decreasing material nonlinearity is dominated by the decrease of dislocation density. In the second phase, the reduction of the dislocation density slows down and the precipitated particles form and grow, which leads to the measured increase in the acoustic nonlinearity parameter. The scanning electron microscopy and hardness measurements support the results obtained from the nonlinear ultrasonic measurements. The monotonic decrease in hardness serves as an indicator for the decrease of the dislocation density, since the curve of the hardness can be described by the dissolution of lath martensite that contains a high dislocation density. Furthermore, the scanning electron microscopy reveals the growth of the precipitated particles as the aging process progresses. The untreated specimen contains small, evenly distributed chromium containing precipitates. SEM images show that the further the aging process advances, the bigger the precipitates are. The acoustic nonlinearity parameter $\beta$ provides an understanding of, and information on, the microstructure and hardness. However, due to the nature of the complex phase transformations that occurs during aging, for an absolute assessment of the microstructre state with the acoustic nonlinearity parameter $\beta$, one needs to develop a quantitative model that describes this evolution process and the corresponding changes in $\beta$.

\section{ACKNOWLEDGMENTS}

This research is being performed using funding received from the DOE Office of Nuclear

Energy's Nuclear Energy University Programs (NEUP). Daniel Marino was supported by the German Academic Exchange Service (DAAD).

\section{REFERENCES}

[1] P. D. Jablonski. 9Cr-1Mo steel material for high temperature application. United States Patent, (Patent N0.2 US 8,317,944 B1), 2012. 
[2] H. K. Danielsen and J. Hald. Influence of Z-phase on long-term creep stability of martensite 9 to 12\% Cr steel. VGB Power Tech, Vol.5: 68-73, 2009.

[3] J. Park, M. Kim, B. Chi,and C. Jang. Correlation of metallurgical analysis and higher harmonic ultrasound response for long term isothermally aged and crept FM steel for USC TPP turbine rotors. NDT\&E International, Vol. 54: 159-165, 2013.

[4] K. H. Matlack, J.Y. Kim, L.J. Jacobs, and Jianmin Qu, Review of second harmonic generation measurement techniques for material state determination in metals," Journal of Nondestructive Evaluation, DOI 10.1007/s 10921-014-0273-5, 2015.

[5] A. Ruiz, N. Ortiz, A. Medina, J.Y. Kim, L. Jacobs. Application of ultrasonic methods for early detection of thermal damage in 2205 duplex stainless steel. NDT\&E International, Vol. 54: 19-26, 2013.

[6] J. S. Valluri, K. Balasubramaniam, R. V. Prakash. Creep damage characterization using non-linear ultrasonic techniques. Acta Materialia, Vol,58: 2079-2090, 2010.

[7] K. H. Matlack, J. J. Wall, J.-Y. Kim, J. Qu, L. J. Jacobs and H.-W. Viehrig. Evaluation of radiation damage using nonlinear ultrasound.Journal of Applied Physics, Vol. 111, 2012.

[8] A. Hikata, B.B. Chick, C. Elbaum. Dislocation contribution to the second harmonic generation of ultrasonic waves. Journal of Applied Physics, Vol.36: 229-236, 1965.

[9] J.H. Cantrell, W.T. Yost. Nonlinear ultrasonic characterization of fatigue microstructures. International Journal of Fatigue, Vol.23(1): 487-490, 2001.

[10] S. Thiele, J.-Y. Kim, J.J. Wall, H.J. Jung, J. Qu, L.J. Jacobs. Nonlinear ultrasonic characterization of precipitation in 17-4PH stainless steel. NDT\&E International, Vol.71: 8-15, 2015.

[11] J. Herrmann, J.-Y. Kim, J. Qu, L. J. Jacobs, J.W. Littles and M. F. Savage. Assessment of material damage in a nickel-base superalloy using nonlinear Rayleigh surface waves. Journal of Applied Physics, Vol. 99, 2006.

[12] J. H. Cantrell and X. G. Zhang. Nonlinear acoustic response from precipitate-matrix misfit in a dislocation network. Journal of Applied Physics, Vol. 84: 5469-5472, 1998.

[13] L. Cipolla, A. Gianfrancesco, D. Venditti, G. Cumino and S. Caminada. Microstructural evolution during long term creep tests of $9 \% \mathrm{Cr}$ steel grades. Eighth International Conference on Creep and Fatigue at Elevated Temperatures, 2007.

[14] J. Hald. Microstructure and long-term creep properties of 9-12\% Cr steels. International Journal of Pressure Vessels and Piping, Vol.85: 30-37, 2008. 
[15] H.K. Danielsen. Z-phase in 9-12\%Cr steels. PhD thesis, Departement of Manufacturing, Technical University of Denmark, 2007.

[16] K. Sawada, K. Miyahara, H. Kushima, K. Kimura. Contribution of microstructural factors to hardness change during creep exposure in mod. $9 \mathrm{Cr}$-1Mo steel. ISIJ International, Vol. 45, 2005.

[17] A. V. Oppenheim and R. W. Schafer. Discrete-time signal processing. Prentice Hall, Upper Saddle River, New Jersey 1999.

[18] D. Torello, S. Thiele, K. H. Matlack, J.-Y. Kim, J. Qu and L. J. Jacobs. Diffraction, attenuation, and source corrections for nonlinear Rayleigh wave ultrasonic measurements. Ultrasonics, Vol. 56: 417-426, 2015.

[19] D. J. Shull, E. E. Kim, M. F. Hamilton and E. A. Zabolotskaya. Diffraction effects in nonlinear Rayleigh wave beams. Journal of the Acoustical Society of America, 1995.

[20] S. V. Walker, J.Y. Kim, J. Qu, L. J. Jacobs. Fatigue damage evaluation in A36 steel using nonlinear Rayleigh surface waves. NDT \& E International, Vol.48: 10-15, 2012.

[21] A. Ruiz, N. Ortiz, H. Carreón, C. Rubio. Utilization of Ultrasonic measurements for determining the variations in microstructure of thermally degraded 2205 duplex stainless steel. Journal of Nondestructive Evaluation, 28(3-4): 131-139, 2009.

[22] W. B. Jones, C. R. Hills, D. H. Polonis. Microstructural evolution of modified 9Cr-1Mo steel. Metallurgical Transactions A, Vol.22: 1049-1058, 1991. 


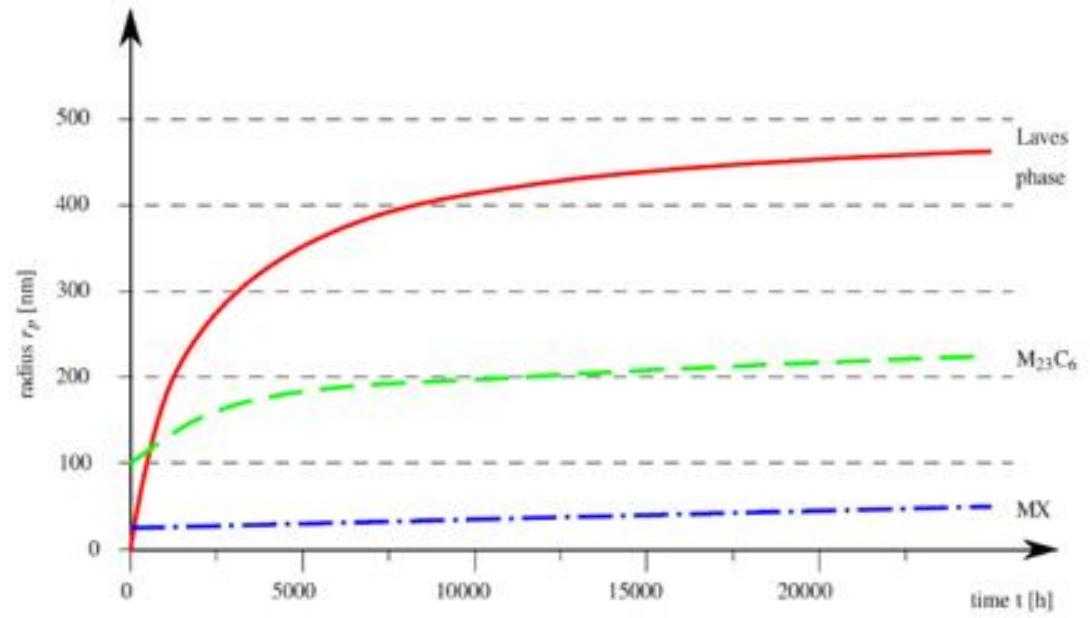

FIGURE 1. Microstructural size evolution of M23C6 and Lave phase in 9\%Cr steel (curves are produced by fitting the experimental data in [13] and [14]).

TABLE 1. Microstructural changes and their effects on $\beta$.

Mechanism

Expected

change in $\beta$

\section{Comments}

\begin{tabular}{|c|c|}
\hline Dislocation Density & $\Lambda^{(\mathrm{d})}$ monotonically decreases \\
\hline Nucleation & Many small precipitates $r_{p}$ \\
\hline Growth & $\begin{array}{l}\text { Precipitation radius } r_{p} \text { and volume fraction } \mathrm{f}_{\mathrm{p}} \\
\text { increase }\end{array}$ \\
\hline Coarsening & $\begin{array}{l}\text { Volume fraction } \mathrm{f}_{\mathrm{p}} \text { remains constant and } \\
\text { precipitates lose their coherency }\end{array}$ \\
\hline
\end{tabular}

Table 2. Chemical composition of 9\%Cr steel

\begin{tabular}{|c|c|c|c|c|c|c|c|}
\hline Element & $\mathrm{C}$ & $\mathrm{Mn}$ & $\mathrm{P}$ & $\mathrm{S}$ & $\mathrm{Si}$ & $\mathrm{Cu}$ & $\mathrm{Ni}$ \\
\hline Percentage (\%) & 0.117 & 0.443 & 0.020 & 0.0005 & 0.330 & 0.090 & 0.153 \\
\hline Element & $\mathrm{Cr}$ & $\mathrm{Mo}$ & $\mathrm{Al}$ & $\mathrm{Nb}$ & $\mathrm{V}$ & $\mathrm{Ti}$ & $\mathrm{N}$ \\
\hline Percentage (\%) & 8.888 & 0.862 & 0.011 & 0.073 & 0.192 & 0.003 & 0.0451 \\
\hline
\end{tabular}




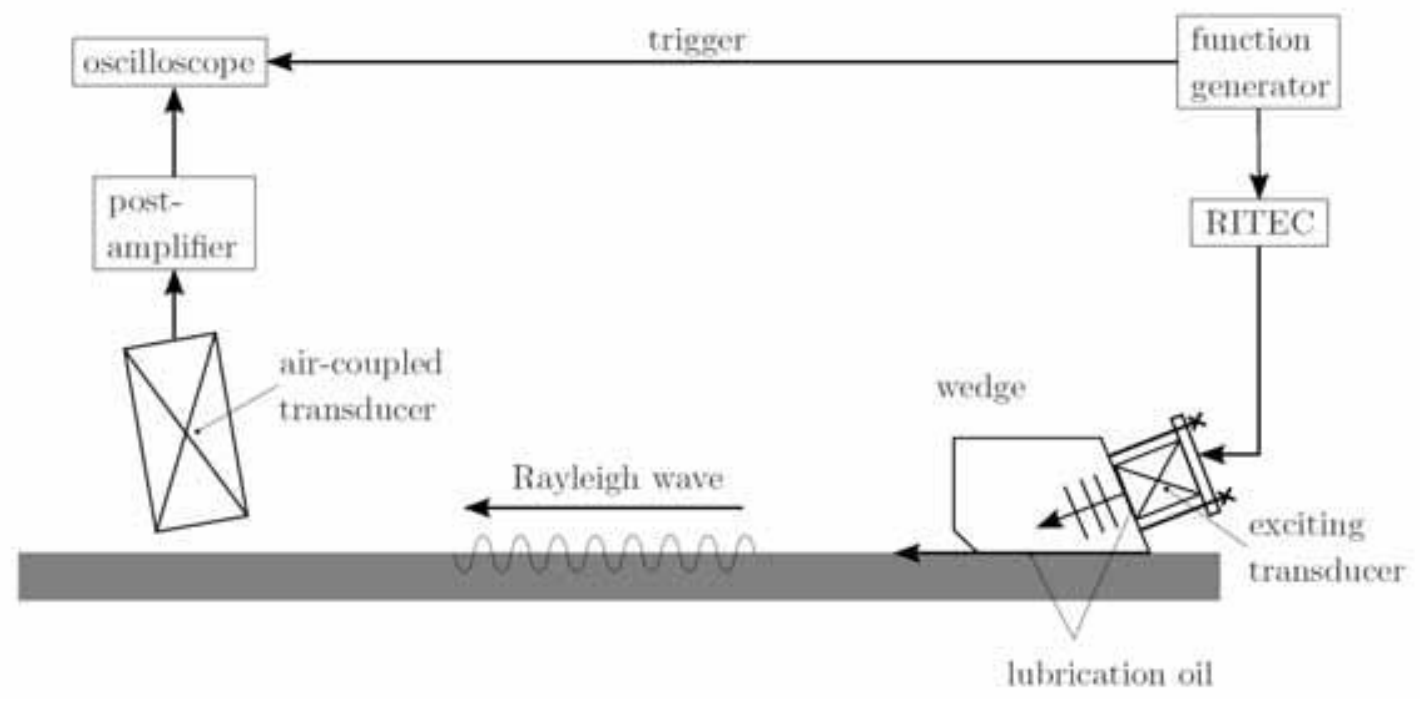

FIGURE2. Experimental setup for nonlinear ultrasound measurements.
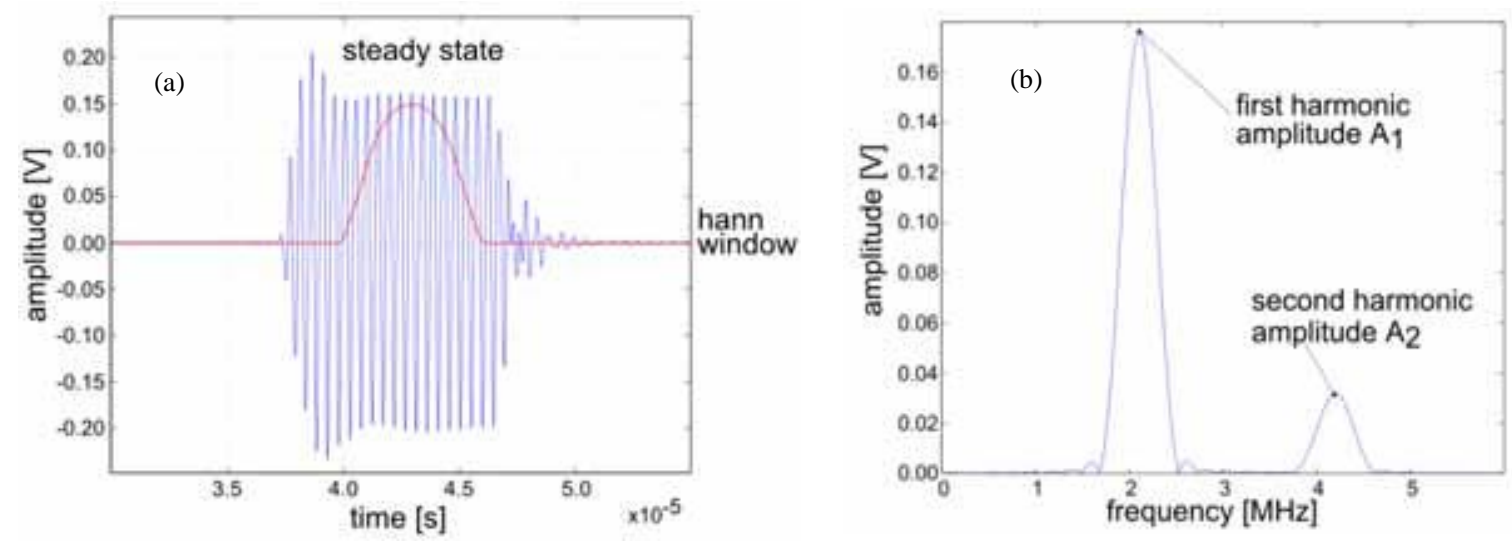

FIGURE3. Typical output signal in and frequency domain with $A_{1}$ and $A_{2}$

(a)

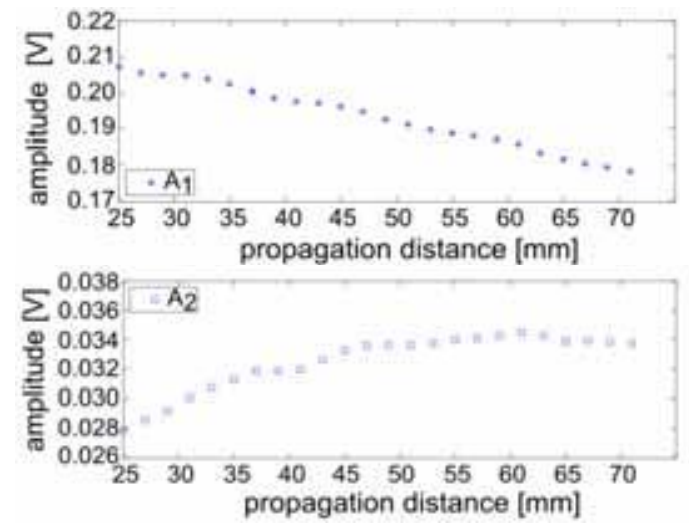

(b)

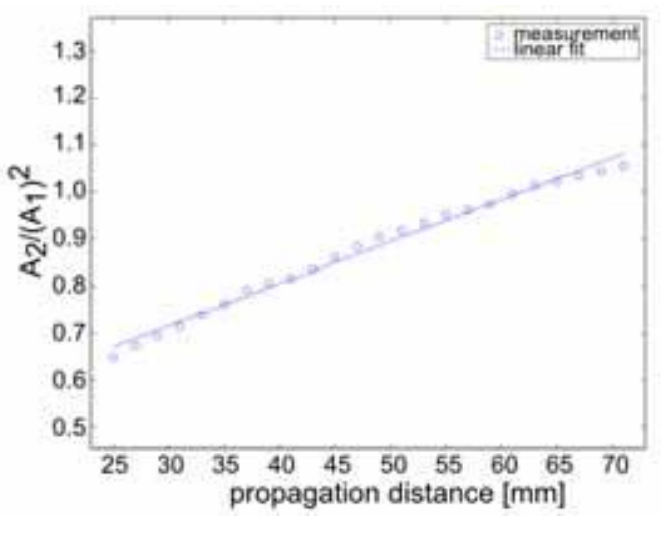


FIGURE 4. Typical results for a measurement with a frequency of $2.1 \mathrm{MHz}$ for sample 1 (untreated sample).
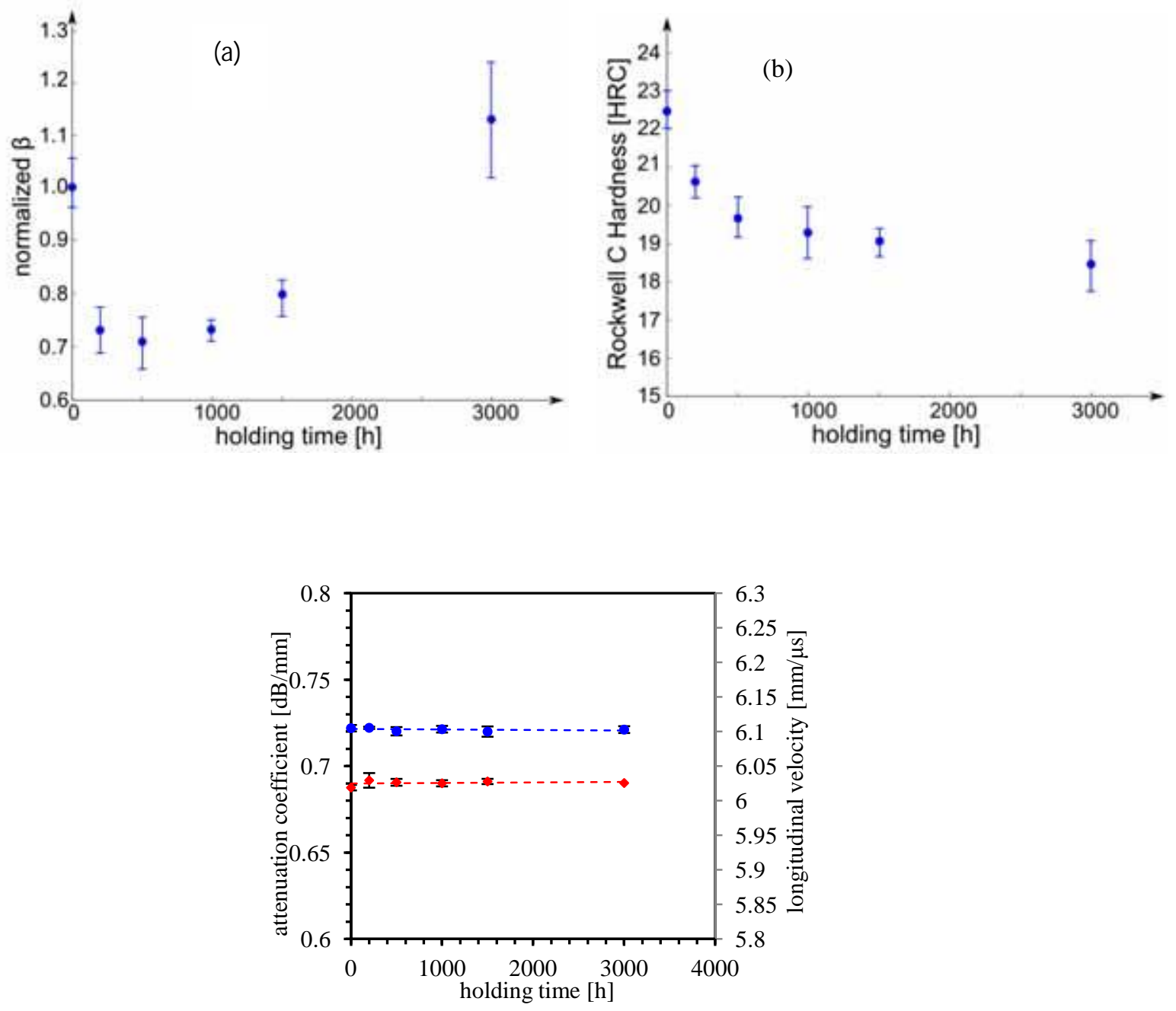

FIGURE 5. Nonlinearity parameter $\beta$ (a) normalized by the mean value of specimen 1 and Rockwell C hardness (b) over holding time.

FIGURE 6. Attenuation and velocity measurements as function of holding time. 


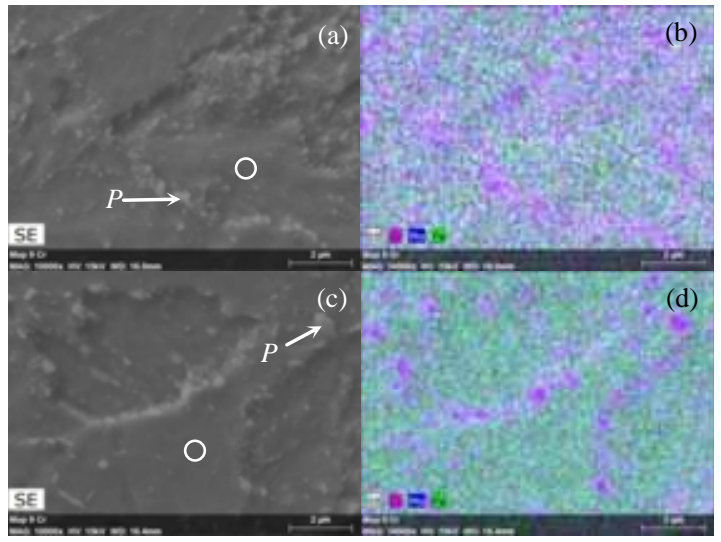

FIGURE 7. Element mapping of specimen with holding time of $0 \mathrm{~h}$ (left) and $3000 \mathrm{~h}$ (right). 
TABLE 3. Element concentrations

Base material $3000 \mathrm{~h}$

\begin{tabular}{c|cc|cc} 
Element & matrix [wt. \%] & particle [wt. \%] & matrix [wt. \%] & particle [wt. \%] \\
\hline Fe & 85.04 & 75.23 & 88.59 & 36.68 \\
$\mathrm{Cr}$ & 7.02 & 11.17 & 7.12 & 47.27 \\
$\mathrm{Mo}$ & 0.43 & 0.99 & 0.4 & 4.53 \\
$\mathrm{C}$ & 4.52 & 9.48 & 3.29 & 8.64
\end{tabular}

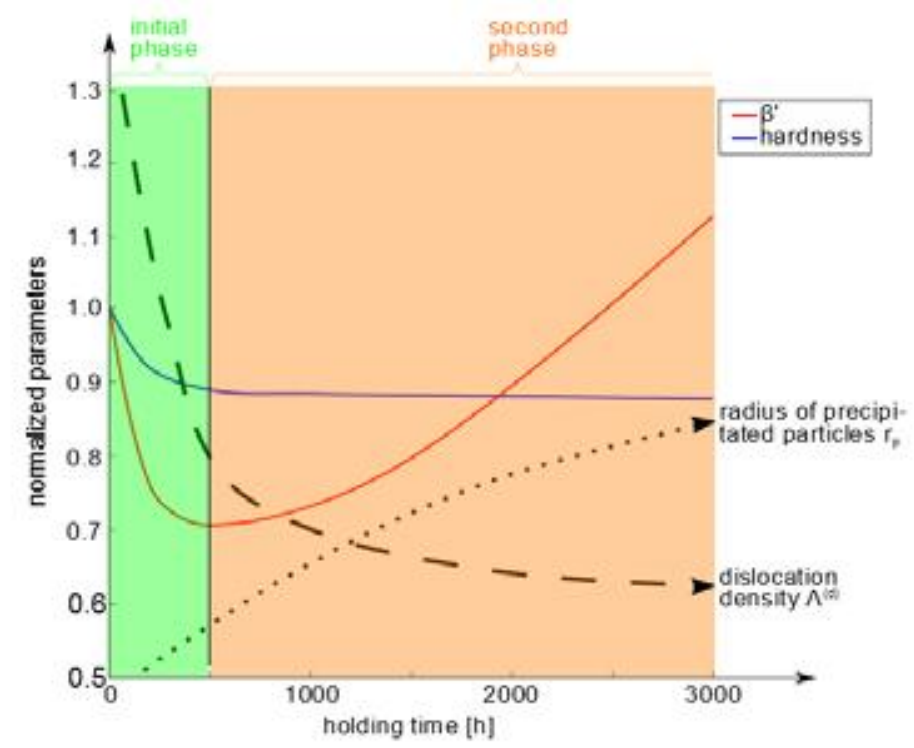

FIGURE 8. Comparison of hardness and ultrasonic results with the qualitative trend of the dislocation density and radius of precipitated particles. 